\title{
Aflibercept in Diabetic Macular Oedema Previously Refractory to Standard Intravitreal Therapy: An Irish Retrospective Study
}

\author{
Clare F. McCloskey · Ann-Marie Mongan · Shivona Chetty • \\ Darren M. J. McAteer · Shauna M. Quinn
}

Received: January 14, 2018 / Published online: March 5, 2018

(C) The Author(s) 2018. This article is an open access publication

\begin{abstract}
Introduction: To determine visual and anatomical outcomes of diabetic macular oedema (DMO) patients in a tertiary centre following conversion to aflibercept having been refractory to previous treatment with bevacizumab/ranibizumab.

Methods: A retrospective case series of patients with a diagnosis of DMO undergoing aflibercept intravitreal therapy for at least 6 months who had previous treatment with three consecutive bevacizumab/ranibizumab injections preswitch. Exclusion criteria included other procedures affecting visual outcome performed within the treatment period. Outcomes measured included visual acuity (VA), central macular thickness (CMT) and injection frequency. Results: Eighteen eyes of 13 patients were included. Mean VA pre-switch was $61.5 \pm 13.8$ letters and CMT was $433.2 \pm 101.4$. Mean number of prior bevacizumab/ranibizumab treatments was $11.3 \pm 7.2$. Mean follow-up post-switch was 22.5 months (SD 7.9). Mean VA
\end{abstract}

Enhanced content To view enhanced content for this article, go to https://doi.org/10.6084/m9.figshare. 5909128.

C. F. McCloskey $(\bowtie) \cdot$ A.-M. Mongan · S. Chetty ·

D. M. J. McAteer · S. M. Quinn

Ophthalmology Department, Sligo University

Hospital, Sligo, Ireland

e-mail: cmccloskey@live.ie improved from baseline by 4.8 letters at 6 months $(p=0.005)$, by 6.1 letters at 12 months $(p=0.006)$, by 7.9 letters $(p=0.004)$ at 18 months and by 6.4 letters $(p=0.1)$ at 24 months. Mean CMT decreased from baseline by $108.6 \mu \mathrm{m}$ at 6 months $(p=0.01), 117.7 \mu \mathrm{m}$ at 12 months ( $p=0.0003), 158.0 \mu \mathrm{m}$ at 18 months $(p=0.005)$ and by $123.3 \mu \mathrm{m}$ at 24 months $(p=0.02)$.

Conclusion: Switching to aflibercept in treatment-resistant DMO produces significant improvements in visual and anatomical outcomes, with eventual maintenance of VA levels.

Keywords: Anti-vascular endothelial growth factor; Diabetic macular oedema; Medical retina

\section{INTRODUCTION}

There were 415 million people worldwide estimated to have diabetes in 2015 [1]. One third of these had signs of diabetic retinopathy defined as visually threatening, severe, non-proliferative or proliferative disease and/or the presence of diabetic macular oedema (DMO) $[2,3]$.

In Ireland, it is estimated that 1 in 10 over the age of 50 have diabetes, equating to $9.5 \%$ or 120,000 people [4]. These numbers are expected to rise significantly in the coming years due to our rapidly ageing population, presenting an increasing clinical challenge. The International Diabetes Federation estimates the number of 
people worldwide with diabetes will reach 642 million by 2040 [1].

Vascular endothelial growth factor (VEGF) is targeted in treatment of DMO by anti-VEGF intravitreal injections. Bevacizumab (Avastin, Roche, UK) and ranibizumab (Lucentis, Novartis, UK) are two anti-VEGF agents used to treat DMO.

Aflibercept (Eylea, Bayer plc, UK) was European Medicines Agency (EMA)-approved for treatment of DMO in June, 2014 [5]. It is a $115-\mathrm{kDa}$ recombinant fusion protein molecule made up of a constant region $(\mathrm{Fc})$ of human immunoglobulin G1 merged with two VEGF receptors (1 and 2 ). It has a higher binding capacity to VEGF-A in relation to bevacizumab and ranibizumab and the advantage of additional binding to VEGF-B and placental growth factor (PIGF), all molecules of which are involved in angiogenesis $[6,7]$.

Subsequent to the phase 2 (DA VINCI) and phase 3 (VISTA and VIVID) clinical trials [6, 7], aflibercept is now established as another option for treatment of DMO. The Diabetic Retinopathy Clinical Research Network (DRCR.net) undertook a randomised control trial (protocol T) comparing each of the three anti-VEGF agents for treatment of DMO. At year 1, they found with mild VA loss, there were comparable improvements in VA among the three agents. Those with worse initial VA had more significant improvements with aflibercept compared to the other two agents [8]. At year 2, aflibercept was still shown to be superior to bevacizumab in those with worse baseline VA; however, the superiority of aflibercept over ranibizumab shown in year 1 did not follow through to year 2 [9].

There are few published studies looking at possible benefits of switching to aflibercept following previous unsuccessful treatment with bevacizumab and ranibizumab. These studies all have a short mean follow-up of 4.6-5 months [10-13]. Therefore, we do not know if the visual and anatomical benefits of aflibercept switch are sustained with longer follow-up.

Our aim is to determine if subjects with DMO non-responsive to previous anti-VEGF therapy would gain any additional benefit in terms of visual and anatomical outcomes when switched to aflibercept and compare our results to other real-life studies.

\section{METHODS}

Our tertiary centre in Sligo, Ireland, introduced intravitreal aflibercept treatment for macular oedema in October, 2014. Consecutive patients with a diagnosis of DMO and treated with aflibercept were identified from a prospectively maintained computerised electronic database (Medisoft Limited, Leeds, UK) and retrospectively analysed. Inclusion criteria were patients with a diagnosis of DMO who had received at least three previous consecutive intravitreal treatments with ranibizumab $(0.5 \mathrm{mg})$, bevacizumab $(1.25 \mathrm{mg})$ or both in the 6 months prior to switching to aflibercept. Minimum follow-up post-aflibercept switch was at least 6 months. Exclusion criteria were any subjects who received procedures affecting possible visual outcomes including phacoemulsification, YAG capsulotomy and corticosteroid treatment (implants/sub-tenon injections) during the treatment period.

The diagnosis of DMO was made based on clinical examination and spectral-domain optical coherence tomography (SDOCT) \pm fundus fluorescein angiography (FFA) findings. Treatment plans, re-treatment plans and injection techniques were devised by the individual treating ophthalmology specialists. The criteria for switching included patients with refractory or persistent DMO defined as no decline, partial resolution or increase in fluid on OCT comparable with fundal examination and declining or no improvement in VA.

Logarithm of the minimal angle of resolution (LogMAR) VAs were recorded at each hospital visit and OCT using Cirrus (Carl Zeiss Meditec, CA, USA) was performed at each clinic visit. Baseline best corrected VA (BCVA) and central macular thickness (CMT) values were recorded from most recent clinic visit prior to switch. Demographic data, treatment intervals and follow-up details were obtained from the electronic patient database. Systemic complications were monitored at each injection visit by 
retrieving any updates in relation to the patients' general health since their previous visit and intra-ocular pressure was measured at each clinic visit following a course of three injections. Ethics approval was obtained from Research Ethics Committee in Sligo University Hospital and the study was executed in agreement with the tenets of the Declaration of Helsinki. This article was based on an analysis of data from a prospectively maintained computerised electronic database and does not involve any studies in humans or animals performed by the authors.

\section{Statistics}

Statistical analysis of visual and anatomical outcomes was performed using Graphical Prism 6 Software (GraphPad Software, Inc., CA, USA). Data were expressed as mean \pm standard deviation (SD), and comparisons were performed using a paired two-sided Student's $t$ test as for related data samples. A test for normality (Shapiro-Wilks test) was performed for each parameter, with $p<0.05$ set as being statistically significant.

\section{RESULTS}

Demographic and baseline characteristics of the DMO cohort are shown in Table 1. Eighteen eyes of 13 patients were included. All patients had received at least three ranibizumab or bevacizumab treatments in the 6 months prior to switch and all patients had at least 6 months follow-up post-switch to be included. Seven eyes (39\%) had been treated with bevacizumab alone, 4 eyes $(22 \%)$ with ranibizumab alone and 7 eyes (39\%) with both. The mean interval between the last ranibizumab/bevacizumab to first aflibercept injection was $66 \pm 19$ days. The mean interval between the first and second aflibercept injection was $49 \pm 27$ days and between the second and third, it was $48 \pm 15$ days. The mean total aflibercept injections until final follow-up was $8.4 \pm 3.9$.
Table 1 Patient demographics and baseline characteristics of the DMO cohort

\begin{tabular}{ll}
\hline Patient demographics & \\
\hline Participants, $n$ & 13 \\
Number of eyes, $n$ & 18 \\
Male, $n$ (\%) & $11(85 \%)$ \\
Right eyes, $n$ (\%) & $8(44 \%)$ \\
Age (years) & $68 \pm 6.6$ \\
Baseline BCVA, number of letters & $61.5 \pm 13.8$ \\
Baseline CMT ( $\mu$ m) & $433.2 \pm 101.4$ \\
Total previous bevacizumab injections, $n$ & $7 \pm 5.6$ \\
Number bevacizumab injections in & $1.8 \pm 1.5$ \\
$\quad$ preceding 6 months, $n$ & \\
Total previous ranibizumab injections, $n$ & $4.3 \pm 4.4$ \\
Number ranibizumab injections in & $1.2 \pm 1.5$ \\
$\quad$ preceding 6 months, $n$ & \\
Total intravitreal injections (IVI) pre- & $11.3 \pm 7.2$ \\
$\quad$ switch, $n$ & \\
Total IVI previous 6 months, $n$ & $22.5 \pm 7.9$ \\
\hline Follow-up (months) & \\
\hline
\end{tabular}

Data expressed as mean $\pm \mathrm{SD}$

$S D$ standard deviation, DMO diabetic macular oedema, $B C V A$ best corrected visual acuity, $C M T$ central macular thickness, $I V I$ intravitreal injections

\section{Visual Outcomes}

Mean BCVA improved by 4.8 letters at 6 months (SD 6.4, $p=0.005$ ), by 6.1 letters at 12 months (SD 7.1, $p=0.006$ ), by 7.9 letters at 18 months (SD 7.0, $p=0.004$ ) and by 6.4 letters at 24 months (SD 10.6, $p=0.1$; Fig. 1).

\section{Anatomical Outcomes}

Mean CMT decreased by $108.6 \mu \mathrm{m}$ at 6 months (SD 160.5, $p=0.01$ ), $117.7 \mu \mathrm{m}$ at 12 months (SD $103.3, p=0.0003), 158.0 \mu \mathrm{m}$ at 18 months (SD $110.3, p=0.005)$ and by $123.3 \mu \mathrm{m}$ at 24 months (SD 104.2, $p=0.02$; Figs. 2, 3). 

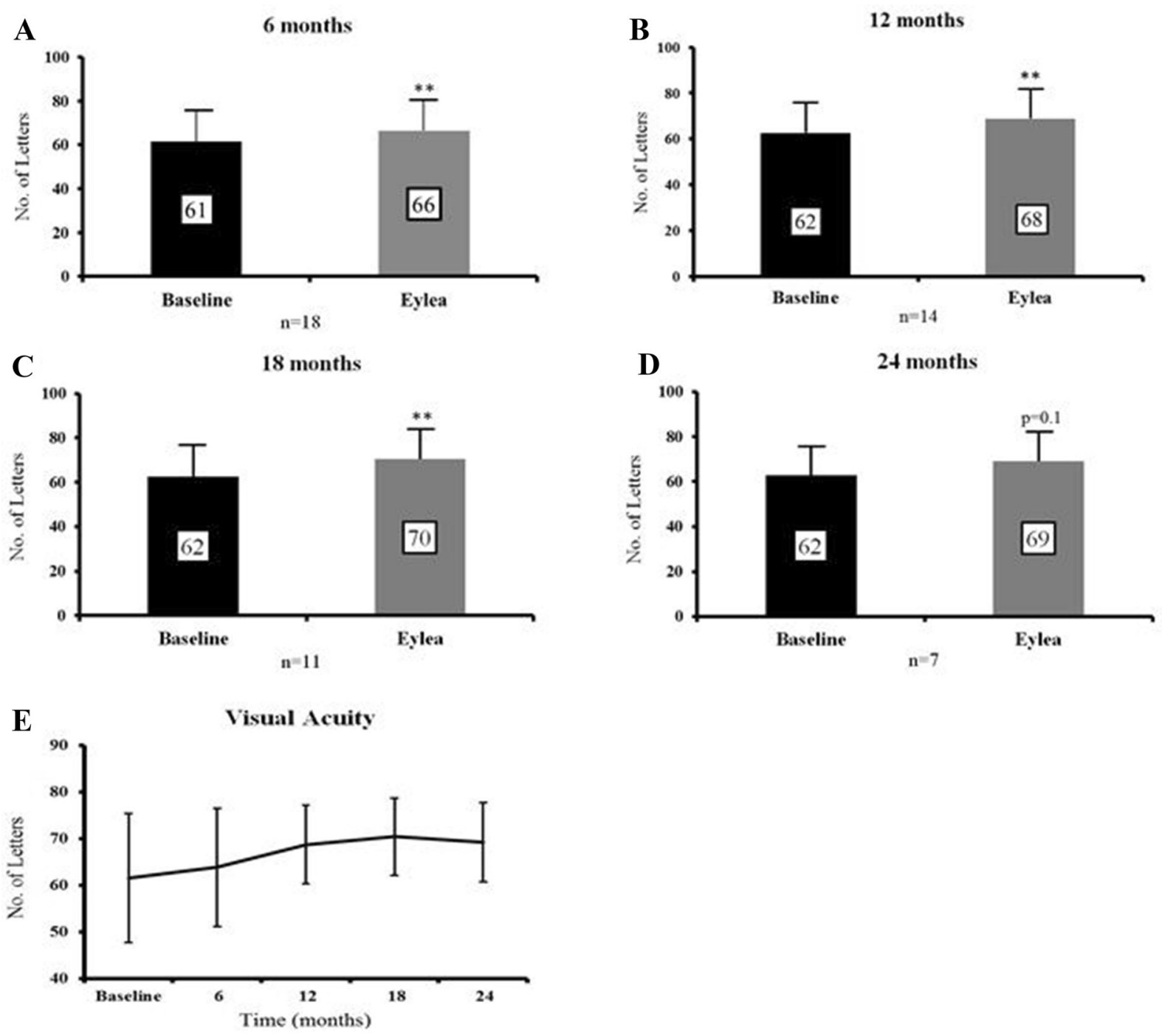

Fig. 1 Mean BCVA improves at each time point compared to baseline. a-d Shows mean visual acuity recorded in LogMAR letters at 6 months (difference of 4.8 letters, $p=0.005$ ), 12 months (difference of 6.1 letters, $p=0.006$ ), 18 months (difference of 7.9 letters, $p=0.004$ ) and 24 months (difference of 6.4 letters, $p=0.1$ ) post-aflibercept (Eylea) switch compared to

\section{Injection Frequency}

The mean total number of injections in the preceding 6 months prior to aflibercept switch was 3 (SD 0). Patients received a mean total of 3.7 injections (SD 0.8, $p=0.004$ ) in the first 6 months post-aflibercept switch and a mean of

baseline visual acuity. e Shows the trend in visual acuity (LogMAR letters) over time. Data are expressed as mean \pm SD. Statistical analysis was performed using the paired two-tailed Student's $t$ test. ${ }^{* *} p<0.01$. BCVA best corrected visual acuity, $\log M A R$ logarithm of the minimal angle of resolution, $S D$ standard deviation

2.2 injections (SD 1.1, $p=0.006$ ) in the subsequent 6 -12-month period. There were no significant systemic (myocardial infarction, transient ischaemic attacks or strokes) or ocular (endophthalmitis, retinal detachment or prolonged elevation in intra-ocular pressure) adverse events during our study period. 

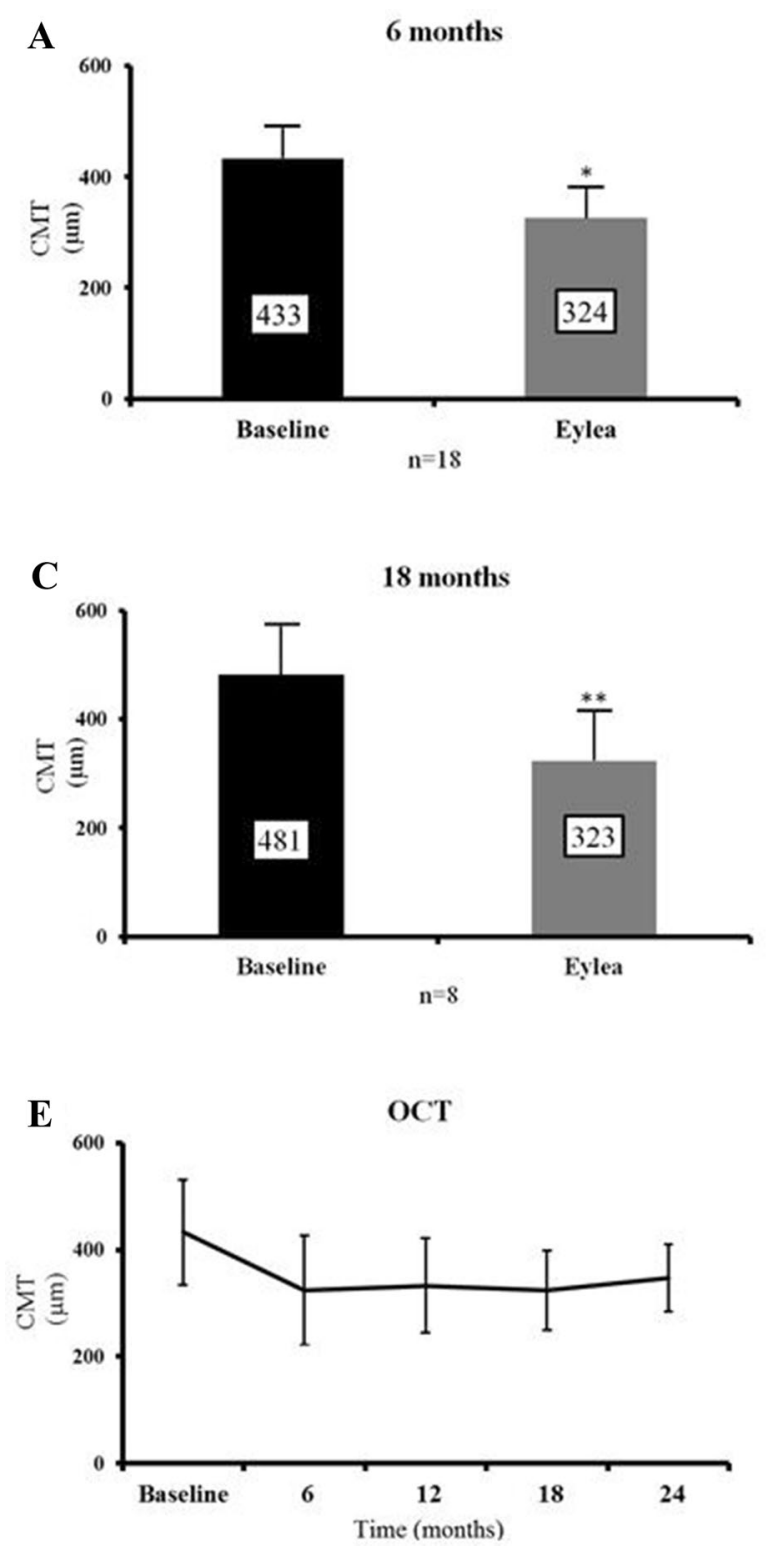

Fig. 2 Mean CMT improves at each time point compared to baseline. a-d Shows mean CMT at 6 months (reduction of $108.6 \mu \mathrm{m}, p=0.01$ ), 12 months (reduction of $117.7 \mu \mathrm{m}, \quad p=0.0003$ ), 18 months (reduction of $158.0 \mu \mathrm{m}, p=0.0048$ ) and 24 months (reduction of $123.3 \mu \mathrm{m}, p=0.02$ ) post-aflibercept (Eylea) switch compared to baseline CMT. e Shows the trend in CMT over

\section{Comparison of Visual and Anatomical} Outcomes in Relation to Pre-switch Visual Acuity

We divided the subjects by baseline VA into two groups; those with better VA pre-switch $(\geq 6 / 12$
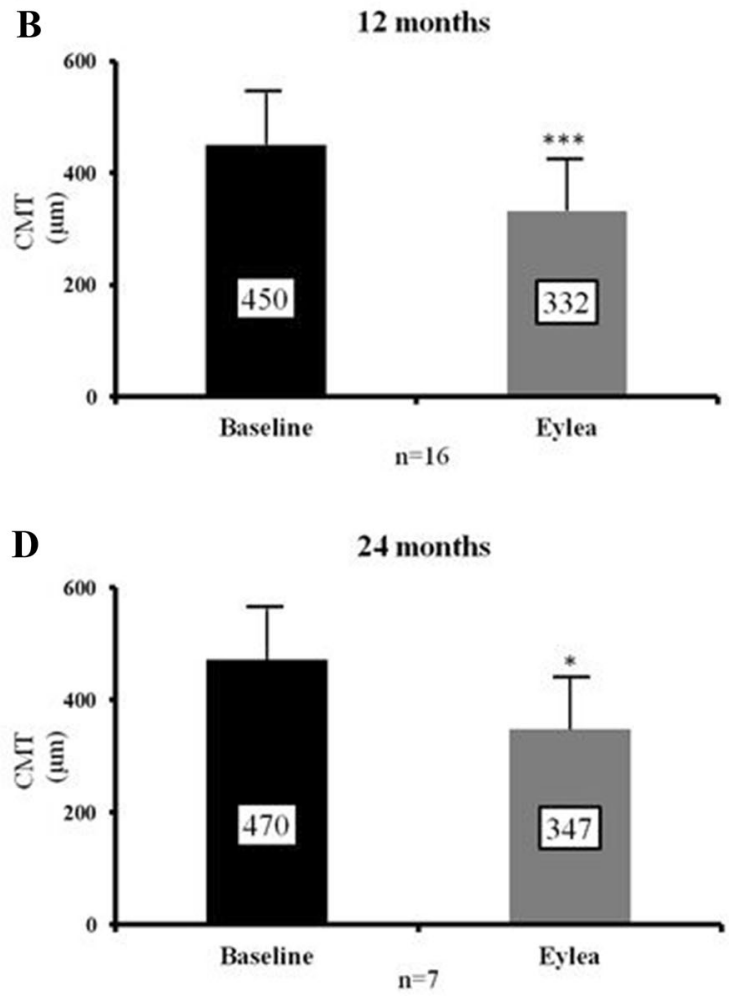

time. Data expressed as mean \pm SD. Statistical analysis was performed using the paired two-tailed Student's $t$ test. ${ }^{*} p<0.05,{ }^{* *} p<0.01,{ }^{* * *} p<0.001$. CMT central macular thickness, $S D$ standard deviation

Snellen or $\geq 0.3$ LogMAR equivalent; group 1 , $n=9)$ and those with worse VA $(<6 / 12$ or $<0.3$ LogMAR; group 2, $n=9$ ). In group 1 , the VA did not demonstrate any improvement. In group 2, the VA showed significant improvements at all time points compared to baseline (Fig. 4). The 


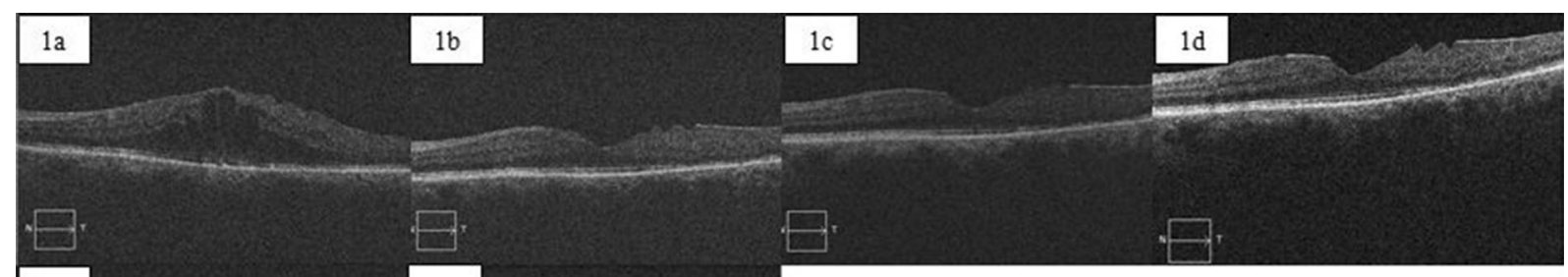

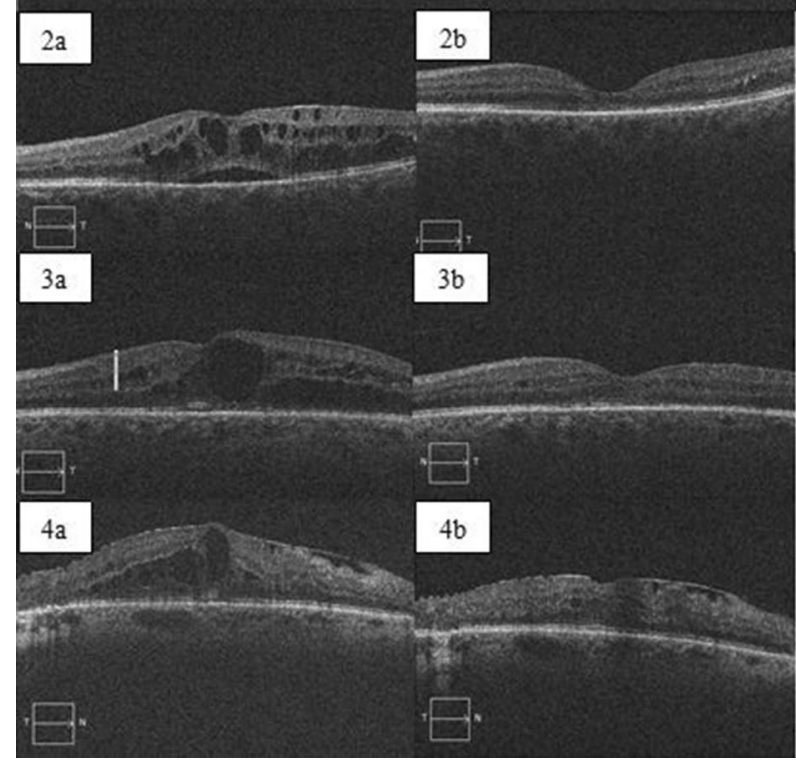

Fig. 3 Serial OCT scans from four different patients undergoing aflibercept treatment injections. 1a Shows IRF prior to commencing aflibercept having undergone 25 previous bevacizumab/ranibizumab injections. $\mathbf{1 b}$ is 6 months post-switch, $\mathbf{1 c}$ is at 12 months and $\mathbf{1 d}$ is at 18 months showing stabilisation after initial resolution. 2 a Shows SRF and cystic IRF pre-switch with seven previous bevacizumab injections and $\mathbf{2} \mathbf{b}$ shows complete resolution

difference in VA between the groups decreases over time (Fig. 4). Of note, there was no difference in the injection interval between the two groups (11.2 versus 11.3 injections).

\section{DISCUSSION}

This study highlights the encouraging visual and anatomical outcomes of patients with DMO refractory to previous intravitreal therapy when treated with aflibercept under "real life" conditions. Patients had statistically significant letter gains at 6,12 and 18 months in relation to baseline VA, with an overall gain of 7.9 letters sustained at 18 months. Patients had statistically significant less intra-retinal and sub-retinal of both SRF and IRF at 6 months. 3a Shows IRF and SRF having had three previous bevacizumab injections and $\mathbf{3 b}$ at 6 months post-aflibercept. 4a Shows IRF with an ERM pre-switch having undergone six previous ranibizumab injections and $\mathbf{4 b}$ showing resolution of IRF, but with continued blunting of the foveal contour at 6 months. IRF intraretinal fluid, $S R F$ subretinal fluid, ERM epiretinal membrane

fluid at six, 12, 18 and 24 months compared to baseline.

In primary treatment of DMO, Nepomuceno et al. showed similar CMT improvements with bevacizumab versus ranibizumab and at some visits (8 and 32 weeks) visual superiority of ranibizumab [14]. Protocol T in primary treatment of DMO at 2 years showed equivalence between bevacizumab versus ranibizumab and aflibercept versus ranibizumab, but superiority of aflibercept over bevacizumab [9].

Lim et al. [11] reviewed 21 diabetic eyes receiving bevacizumab/ranibizumab treatment in Singapore and Massachusetts, USA, and found after aflibercept switch, a mean decrease of $107.0 \mu \mathrm{m}$ in CMT at 5 months, similar to our 

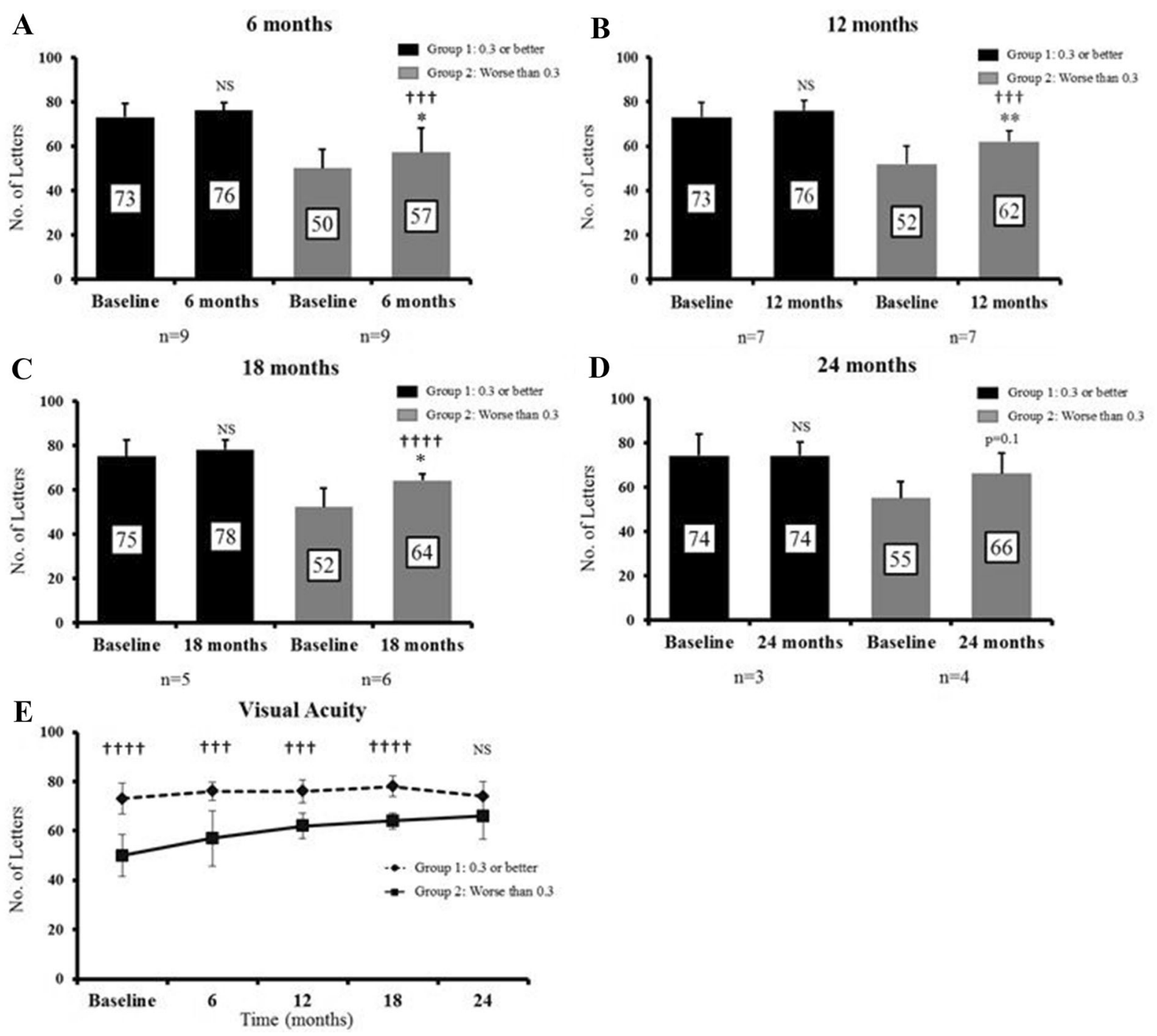

Fig. 4 Good versus poor VA at each time point compared to baseline and each other. Baseline VA was divided into two groups; group 1: VA 0.3 LogMAR or better and group 2: worse than $0.3 \operatorname{LogMAR}$. At each time point, VAs in each group were compared to their individual baselines and directly with each other. a-d Shows non-significant improvements in VA in group 1 at all time points. a Shows statistical improvements in VA at 6 months in group 2 (difference of 6.7 letters, $p=0.02$ ) compared with baseline. At 6 months, there is a statistically significant difference between the good- and poor-VA groups $(p=0.0002)$. b, c Show statistically significant change in $\mathrm{VA}$ at 12 and 18 months in group 2 compared to baseline (difference of 10 letters, $p=0.008$ and 11.5 letters, $p=0.01$, respectively). At 12 and 18 months, there are statistically significant differences between the good- and

poor-VA groups; $p=0.0002$ and $p<0.00001$, respectively. d Shows non-significant improvements in group 2 at 24 months $(p=0.1)$. e Demonstrates the VA trend over the 24-month period showing the difference between the two groups narrowing with time. Data expressed as mean \pm SD. Comparison between baseline VA and VA at each time point performed using the paired two-tailed Student's $t$ test, ${ }^{*} p<0.05,{ }^{* *} p<0.01$. Comparison between group 1/group 2 with baseline performed using the paired two-tailed Student's $t$ test, ${ }^{\dagger+\dagger} p<0.001$, $p<0.0001$. The unpaired $t$ test was used for comparison of group 1 versus group 2. NS non-significant, $\log M A R$ logarithm of the minimal angle of resolution, $V A$ visual acuity, $S D$ standard deviation 
decrease of $108.6 \mu \mathrm{m}$ at 6 months. LogMAR at 5 months improved by 0.05 , and our study patients demonstrated an improvement of 0.10 LogMAR at 6 months [11]. Rahimy et al. [10] assessed 50 DMO eyes in Pennsylvania, USA, that received a mean of 13.7 prior bevacizumab/ ranibizumab injections prior to switch and found after a 4.6-month follow-up period, a change in VA of -0.14 LogMAR and reduction of $184 \mu \mathrm{m}$ compared to baseline CMT [10]. Shah and Heier [12] found following a mean of 16 prior bevacizumab/ranibizumab injections borderline significant VA improvements and significant anatomical improvements after 2 months of aflibercept [12].

Possible reasons for the significant anatomical improvement with aflibercept are due to its different pharmacokinetic and pharmacodynamic properties. Aflibercept has a higher binding affinity to VEGF-A, leading to possibly sustained VEGF-A inhibition [10]. Furthermore, it can bind to VEGF-B and PIGF which are two additional growth factors released during angiogenesis [10].

Another reason for the additional clinical response with aflibercept is tachyphylaxis, which patients may eventually succumb to, with repetitive treatment injections of bevacizumab/ranibizumab $[10,15,16]$. This has been established in many studies looking specifically at age-related macular degeneration (AMD). The mechanism is multifactorial, involving macrophage up-regulation of VEGF, varying surface receptor expression, changes in pharmacokinetics and development of neutralising antibodies as part of a systemic immune response [10]. Forooghian et al. [15] retrospectively looked at 59 patients treated with bevacizumab $(1.25 \mathrm{mg})$ for exudative AMD over a 14-month period and defined tachyphylaxis as a loss of therapeutic response to intravitreal bevacizumab approximately 28 days after administration in an eye which had previously responded to treatment. They identified 5 patients ( 6 eyes) who developed tachyphylaxis within a median time of 100 weeks (range 31-128 weeks) and the median number of injections to establish tachyphylaxis was 8 injections (range 5-10). Therapeutic response did not improve with a higher dosing regimen
(2.50 mg) [15]. Eghoj and Sørensen [16] looked at 1076 exudative AMD eyes (976 patients) and the potential tachyphylaxis effect with intravitreal ranibizumab. They found a tachyphylaxis rate of $2 \%$ (20 patients) in their cohort [16].

Overall, we found mild improvements in VA which did not match the dramatic anatomical improvements. Possible explanations for this could be the long-term damage to the minute retinal interconnections at the macula as a result of the persistent fluid.

We divided our patients in terms of baseline VA, dividing the cohort into those with better versus worse VA. Those with poor vision had statistically significant improvements in VA at each time point compared to baseline. The difference in VA between the two groups narrowed with time, although the change in LogMAR letters at each time point was not statistically significant. Protocol $\mathrm{T}$ described at 1 year that those with worse initial VA did better with aflibercept than those with better baseline VA [8]. In our study, subjects who began aflibercept treatment with worse VA had a trend toward better improvements in vision compared to those who began treatment with good VA. This was not statistically significant possibly due to our small study population. Importantly, in our cohort, there was no difference in the number of injections between the two groups, indicating that subjects with worse VA still had improvements with the same frequency of treatment as those with good VA.

The mean number of bevacizumab/ranibizumab injections in the 6 months prior to switch was 3 (SD 0), representing injections administered weekly for eight weeks. Our centre began to use aflibercept in October, 2014 for select patients before the indication was expanded following protocol T (year 1) in 2015. Prior to aflibercept, the only available antiVEGF treatment was bevacizumab and ranibizumab. Initially, patients were commenced on six-week regimens and clinical response was monitored. If patients did not respond or plateaued, the intensity of treatment was reduced and subsequently patients were classified as refractory or partial responders prior to the era of aflibercept. This explains why patients may not have been receiving six-week treatment 
injections in the 6 months prior to aflibercept in our centre. The introduction of aflibercept now offers these refractory and partial responders another option for further gains in VA.

Subjects received a mean of 3.7 (SD 0.8) aflibercept injections in the first 6 months of treatment. This is less than the recommended five injections every 4 weeks within the first 6 months. Rahimy et al. [10] treated patients with an injection frequency of 4.1 injections within 4.6 months equating to an aflibercept injection every 31 days. Despite more frequent injections in their study, our cohort still had comparable visual and anatomical improvements [10]. A postulated advantage of aflibercept is the longer half-life and therefore potential reduction in injection frequency [17]. After 6 months of aflibercept, our cohort showed a significant trend towards reduction in injection frequency.

This study adds to the limited evidence for aflibercept switch in persistent DMO patients as there are few published studies to our knowledge. The advantages of our study include the fact that the data is from a real-life setting which best reflects daily clinical practice. Our longer follow-up period averaging 22.5 months, when compared to other published studies which only have between 4.6 and 5 months follow-up, shows that improvements in VA and CMT are sustained following aflibercept switch.

Limitations of our study include the retrospective study design, but this best reflects the "real-life" setting. Our study has a small sample size, but this highlights novel experience with aflibercept. In AMD switch studies, it is suggested that such dramatic anatomical improvement with aflibercept may be due to the "loading dose effect" and overcoming tachyphylaxis associated with previous anti-VEGF therapies [18-20]. However, our study shows sustained visual and anatomical improvements long after the "loading dose effect" $[19,20]$. A control group for comparison in a future randomised control trial would be essential for further evaluation of this effect.

The improvements in VA and CMT in our cohort could be due to the fact that patients were receiving eight weekly injections rather than a 4-6 weekly intensive regimen of bevacizumab/ranibizumab prior to switch. However, again, this represents the "real-life" clinical setting and increasing demands of a medical retina service.

\section{CONCLUSIONS}

In conclusion, our study highlights the benefits of an additional anti-VEGF treatment option in terms of visual and anatomical improvements. It is advantageous to know that there are further possible treatment strategies for patients who may have been previously considered refractory or non-responsive to initial intravitreal therapy.

\section{ACKNOWLEDGEMENTS}

Funding. No funding or sponsorship was received for this study or publication of this article. The article processing charges were funded by the authors.

Authorship. All named authors meet the International Committee of Medical Journal Editors (ICMJE) criteria for authorship for this article, take responsibility for the integrity of the work as a whole and have given their approval for this version to be published.

Contributions. All authors have read and approved this manuscript. Clare F. McCloskey: design and conduct of the study; compilation, management, analysis, and interpretation of the data; preparation, assessment and approval of the document. Ann-Marie Mongan: statistical analysis; interpretation; assessment and approval of the document. Shivona Chetty and Darren M. J. McAteer: assessment and approval of the document. Shauna M. Quinn: design and conduct of the study; preparation, assessment and approval of the document; supervision. Presented at the Irish College of Ophthalmologists annual meeting in May 2017.

Presentation. Clare F. McCloskey, AnnMarie Mongan, Shivona Chetty, Darren M. J. McAteer, Shauna M. Quinn: 'Aflibercept in 
neovascular age related macular degeneration previously refractory to standard intravitreal therapy: An Irish perspective to compare against international trends' was presented at EVER in September, 2017.

Disclosures. Clare F. McCloskey, Ann-Marie Mongan, Shivona Chetty, Darren M. J. McAteer and Shauna M. Quinn have nothing to disclose.

Compliance with Ethics Guidelines. Ethics approval was obtained from Research Ethics Committee in Sligo University Hospital and the study was executed in agreement with the tenets of the Declaration of Helsinki. This article was based on an analysis of data from a prospectively maintained computerised electronic database and does not involve any studies in humans or animals performed by the authors.

Data Availability. The datasets generated during and/or analysed during the current study are available from the corresponding author on reasonable request.

Open Access. This article is distributed under the terms of the Creative Commons Attribution-NonCommercial 4.0 International License (http://creativecommons.org/licenses/ by-nc/4.0/), which permits any noncommercial use, distribution, and reproduction in any medium, provided you give appropriate credit to the original author(s) and the source, provide a link to the Creative Commons license, and indicate if changes were made.

\section{REFERENCES}

1. IDF diabetes atlas, International Diabetes Federation, 7th ed. pp.12-13. http://www.diabetesatlas. org. Accessed 3 Mar 2017.

2. Yau J, Rogers S, Kawasaki R, Lamoureux E, Kowalski J, Bek T, et al. Global prevalence and major risk factors of diabetic retinopathy. Diabetes Care. 2012;35:556-64.

3. Lee R, Wong T, Sabanayagam C. Epidemiology of diabetic retinopathy, diabetic macular edema and related vision loss. Eye Vision. 2015;2:1-25.
4. Leahy S, O'Halloran A, O'Leary N, Healy M, McCormack M, Kenny R, et al. Prevalence and correlates of diagnosed and undiagnosed type 2 diabetes mellitus and pre-diabetes in older adults: findings from the Irish Longitudinal Study on Ageing (TILDA). Diabetes Res Clin Pract. 2015;110:241-9.

5. European Medicines Agency-Find medicineEylea. Ema.europa.eu. 2017. http://www.ema. europa.eu/ema/index.jsp?curl=pages/medicines/ human/medicines/002392/human_med_001598. jsp\&mid=WC0b01ac058001d124. Accessed 3 Mar 2017.

6. Do D, Schmidt-Erfurth U, Gonzalez V, Gordon C, Tolentino M, Berliner A, et al. The DA VINCI study: phase 2 primary results of VEGF trap-eye in patients with diabetic macular edema. Ophthalmology. 2011;118:1819-26.

7. Brown D, Schmidt-Erfurth U, Do D, Holz F, Boyer D, Midena E, et al. Intravitreal aflibercept for diabetic macular edema: 100-week results from the VISTA and VIVID studies. Ophthalmology. 2015;122:2044-52.

8. Diabetic Retinopathy Clinical Research Network, Wells J, Glassman A, Ayala A, Jampol LM, Aiello LP, Antoszyk AN, et al. Aflibercept, bevacizumab, or ranibizumab for diabetic macular edema. N Engl J Med. 2015;372:1193-203.

9. Wells J, Glassman A, Ayala A, Jampol LM, Bressler NM, Bressler SB, et al. Diabetic retinopathy clinical research network. aflibercept, bevacizumab, or ranibizumab for diabetic macular edema. Ophthalmology. 2016;123:1351-9.

10. Rahimy E, Shahlaee A, Khan M, et al. Conversion to aflibercept after prior anti-VEGF therapy for persistent diabetic macular edema. Am J Ophthalmol. 2016;164(118-127):e2.

11. Lim L, Papakostas T, Kim L, Ying G, Maguire J, Ho $A$, et al. Conversion to aflibercept for diabetic macular edema unresponsive to ranibizumab or bevacizumab. Clin Ophthalmol. 2015;9:1715-8.

12. Shah C, Heier J. Aflibercept for diabetic macular edema in eyes previously treated with ranibizumab and/or bevacizumab may further improve macular thickness. Ophthalmic Surg Lasers Imaging Retina. 2016;47:836-9.

13. Bahrami B, Hong T, Zhu M, Schlub TE, Chang A. Switching therapy from bevacizumab to aflibercept for the management of persistent diabetic macular edema. Graefe's Arch Clin Exp Ophthalmol. 2017;255(6):1133-40. 
14. Nepomuceno A, Takaki E, Paes de Almeida F, Peroni $\mathrm{R}$, Cardillo J, Siqueira R, et al. A Prospective randomized trial of intravitreal bevacizumab versus ranibizumab for the management of diabetic macular edema. Am J Ophthalmol. 2013;156(502-510): e2.

15. Forooghian F, Cukras C, Meyerle C, Chew E, Wong $\mathrm{W}$. Tachyphylaxis after intravitreal bevacizumab for exudative age-related macular degeneration. Retina. 2009;29:723-31.

16. Eghøj M, Sørensen T. Tachyphylaxis during treatment of exudative age-related macular degeneration with ranibizumab. Br J Ophthalmol. 2011;96: 21-3.

17. Thomas M, Mousa SS, Mousa SA. Comparative effectiveness of aflibercept for the treatment of patients with neovascular age-related macular degeneration. Clin Ophthalmol. 2013;7:495-501.
18. Spooner K, Hong T, Wijeyakumar W, Chang A. Switching to aflibercept among patients with treatment-resistant neovascular age-related macular degeneration: a systematic review with meta-analysis. Clin Ophthalmol. 2017;11:161-77.

19. Menon G, Chandran M, Sivaprasad S, Chavan R, Narendran N, Yang Y. Is it necessary to use three mandatory loading doses when commencing therapy for neovascular age-related macular degeneration using bevacizumab? (BeMOc Trial). Eye. 2013;27:959-63.

20. Gharbiya M, Iannetti L, Parisi F, De Vico U, Mungo M, Marenco M. Visual and anatomical outcomes of intravitreal aflibercept for treatment-resistant neovascular age-related macular degeneration. BioMed Res Int. 2014;2014:1-7. 